\title{
Erratum to: Deformation-obstruction theory for complexes via Atiyah and Kodaira-Spencer classes
}

\author{
Daniel Huybrechts • Richard P. Thomas
}

Published online: 11 January 2014

(C) Springer-Verlag Berlin Heidelberg 2014

\section{Erratum to: Math. Ann. (2010) 346:545-569 DOI 10.1007/s00208-009-0397-6}

Fabian Langholf and Pierrick Bousseau pointed out that Lemma 2.2 in the Original publication requires $X$ and $A$ to be flat over the base $B$. For instance the sequence (2.4) in Proof of Lemma 2.2 is not in general exact without this assumption.

After inserting the assumption of flatness of $X_{0}, X$ and $A$ over $B$, Sects. 2 and 3 can be left unchanged. Applied to $B=\operatorname{Spec} k$ with $k$ a field, this is enough to produce virtual cycles in Sect. 4. But to prove their deformation invariance, we need to work with a relative moduli space $\mathcal{M} / B$ whose flatness over $B$ cannot be assumed. In fact, in the Behrend-Fantechi relative obstruction theory of Sect. 4 , flatness of $X$ and $X_{0}$ over the base does not hold in general, so we prefer to proceed as follows. ${ }^{1}$

- Throughout Sects. 2 and 3 we set $B=\operatorname{Spec} k$ with $k$ a field. All necessary flatness assumptions are then automatic. Thus, without further modifications, the paper defines the absolute, rather than relative, truncated Atiyah class $A\left(E_{0}\right)$ and the absolute truncated Kodaira-Spencer class $\kappa\left(X_{0} / X\right)$. Then Corollary 3.4 proves that their product

\footnotetext{
1 Alternatively the reader could consult arXiv:0805.3527v2, which is a corrected version of the paper in which these modifications have been enacted.
}

The online version of the original article can be found under doi:10.1007/s00208-009-0397-6.

D. Huybrechts $(\bowtie)$

Mathematisches Institut, Universität Bonn,

Endenicher Allee 60, 53115 Bonn, Germany

e-mail: huybrech@math.uni-bonn.de

R. P. Thomas

Department of Mathematics, Imperial College,

London SW7 2AZ, UK

e-mail: rpwt@ic.ac.uk 


$$
\left(\operatorname{id}_{E_{0}} \otimes \kappa\left(X_{0} / X\right)\right) \circ A\left(E_{0}\right) \in \operatorname{Ext}_{X_{0}}^{2}\left(E_{0}, E_{0} \otimes I\right)
$$

is the obstruction to deforming a perfect complex $E_{0}$ from $X_{0}$ to its thickening $X$.

- To define relative versions of these objects we first need to define the relative truncated cotangent complex. To do this we embed $X_{0} \subset X$ as follows:

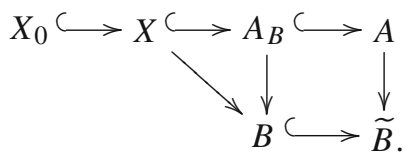

Here $\widetilde{B}$ and $A \rightarrow \widetilde{B}$ are both smooth, and the square is Cartesian (i.e. $A_{B}=A \times{ }_{B} \widetilde{B}$ ). Thus $A$ and $A_{B} \rightarrow B$ are also smooth. Letting $J_{0 B}$ denote the ideal sheaf of $X_{0} \subset A_{B}$ we get the natural commutative diagram

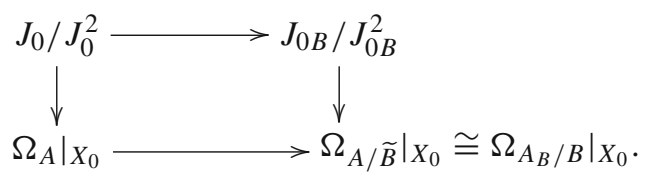

The vertical 2-complex on the left is $\mathbb{L}_{X_{0}}$ and we define $\mathbb{L}_{X_{0} / B}$ to be the vertical 2 -term complex on the right, giving the projection

$$
\mathbb{L}_{X_{0}} \rightarrow \mathbb{L}_{X_{0} / B}
$$

Applied to the Atiyah class $A\left(E_{0}\right) \in \operatorname{Ext}_{X_{0}}^{1}\left(E_{0}, E_{0} \otimes \mathbb{L}_{X_{0}}\right)$ this defines a relative Atiyah class $A\left(E_{0} / B\right) \in \operatorname{Ext}_{X_{0}}^{1}\left(E_{0}, E_{0} \otimes \mathbb{L}_{X_{0} / B}\right)$.

The natural map $J_{0} / J_{0}^{2} \rightarrow I$ factors through $J_{0 B} / J_{0 B}^{2} \rightarrow I$. The former defines the Kodaira-Spencer class $\kappa\left(X_{0} / X\right): \mathbb{L}_{X_{0}} \rightarrow I[1]$, so using the latter to define the relative Kodaira-Spencer class $\kappa\left(X_{0} / X / B\right): \mathbb{L}_{X_{0} / B} \rightarrow I[1]$ we see they commute with the projection (1.2).

Thus the product of the relative Atiyah class and the relative Kodaira-Spencer class equals the product (1.1) of their absolute versions:

$$
\left(\operatorname{id}_{E_{0}} \otimes \kappa\left(X_{0} / X / B\right)\right) \circ A\left(E_{0} / B\right)=\left(\operatorname{id}_{E_{0}} \otimes \kappa\left(X_{0} / X\right)\right) \circ A\left(E_{0}\right) \in \operatorname{Ext}_{X_{0}}^{2}\left(E_{0}, E_{0} \otimes I\right) .
$$

- Finally, in Sect. 4, we project the Atiyah class $A(\mathbb{E}) \in \operatorname{Ext}_{X \times_{B} \mathcal{M}}^{1}\left(\mathbb{E}, \mathbb{E} \otimes \mathbb{L}_{X \times \times_{B} \mathcal{M}}\right)$ of the universal sheaf $\mathbb{E}$ by $\mathbb{L}_{X \times_{B} \mathcal{M}} \rightarrow \mathbb{L}_{X \times_{B} \mathcal{M} / X}=\pi_{\mathcal{M}}^{*} \mathbb{L}_{\mathcal{M} / B}$ to give Eq. 4.2:

$$
\pi_{\mathcal{M} *}\left(\mathcal{H o m}(\mathbb{E}, \mathbb{E})_{0} \otimes \pi_{X}^{*} \omega_{X / B}\right)[n-1] \longrightarrow \mathbb{L}_{\mathcal{M} / B}
$$

The proof that this is a perfect obstruction theory proceeds without change using the relative classes defined above. As in Eq. 4.5 we end up with an obstruction class 
expressed as a product of a relative truncated Atiyah class $A\left(\bar{f}^{*} \mathbb{E} / X\right)$ and a relative truncated Kodaira-Spencer class $\kappa\left(X \times{ }_{B} S_{0} / X \times{ }_{B} S / X\right)=\bar{\pi}^{*} \kappa\left(S_{0} / S / B\right)$.

This equals the product of the corresponding absolute classes by (1.3). By Corollary 3.4 , this equals the obstruction to deforming $\bar{f}^{*} \mathbb{E}$ from $X \times{ }_{B} S_{0}$ to $X \times_{B} S$. Since such deformations are in one-to-one correspondence with extensions from $S_{0}$ to $S$ of the $B$-map $f$, the proof concludes just as in Original publication. 